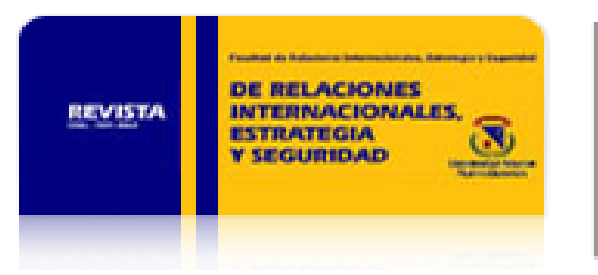

Revista de Relaciones Internacionales, Estrategia y Seguridad

ISSN: 1909-3063

cinuv.relinternal@unimilitar.edu.co

Universidad Militar Nueva Granada

Colombia

Escobar, Natalia; Vega, Julieth

ARMAS BIOLÓGICAS ¿UNA AMENAZA TERRORISTA PARA COLOMBIA?

Revista de Relaciones Internacionales, Estrategia y Seguridad, vol. 3, núm. 2, julio-diciembre, 2008,

pp. $135-148$

Universidad Militar Nueva Granada

Bogotá, Colombia

Disponible en: http://www.redalyc.org/articulo.oa?id=92712967007

- Cómo citar el artículo

- Número completo

- Más información del artículo

- Página de la revista en redalyc.org

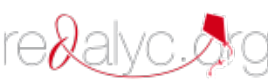

Sistema de Información Científica

Red de Revistas Científicas de América Latina, el Caribe, España y Portugal

Proyecto académico sin fines de lucro, desarrollado bajo la iniciativa de acceso abierto 
rev.relac.int.estrateg.segur.3(2):135-148,2008

\title{
ARMAS BIOLÓGICAS \\ ¿UNA AMENAZA TERRORISTA PARA COLOMBIA?
}

\author{
Natalia Escobar* \\ Julieth Vega**
}

\section{RESUMEN}

Las armas biológicas, naturales y o sintéticas, son productos generados a partir de algún organismo vivo o derivado de su manipulación físico-química.

Hasta hace poco tiempo para los colombianos, los ataques mercenarios con microorganismos no generaban motivo de preocupación, puesto que históricamente se han desarrollado en latitudes distantes a América latina. En este sentido, entre los estados y comunidades comprometidos en prolongados conflictos, es paralela la presencia del terrorismo, situación que estimula una permanente sofisticación de las técnicas de ataque, lo que alienta dentro de sus cadenas de producción, alcanzar niveles más complejos de manipulación tecnológica en este tipo de armas.

\footnotetext{
* Especialista en Microbiología médica y Docencia Universitaria, Docente-investigadora. Universidad Militar Nueva Granada, Universidad de Cundinamarca. E-mail: naescodos21@yahoo.com,

** Joven Investigadora de la Universidad Militar Nueva Granada, Profesional en Relaciones Internacionales y Estudios Políticos de la Universidad Militar Nueva Granada.
} 
No obstante, en el país y particularmente durante las tres ultimas décadas, la violencia interna, la presencia de la narco-guerrilla y el narco-paramilitarismo generaron distintas formas de terrorismo, siendo esta abominable modalidad usada para perpetrar secuestros masivos, realizar atentados a instituciones, vulnerar la infraestructura petrolera y ejecutar tomas de municipios, estableciendo desde esas acciones la cíclica alteración del orden público, hoy neutralizado por las estrategias políticas de la seguridad democrática.

Mundialmente el terrorismo viene incorporado en sus incursiones el uso de armas biológicas, lo cual determina para nuestro sistema de vigilancia y defensa nacional, diseñar y poner en ejecución, oportunamente protocolos y esquemas de control y prevención contra este tipo de ataques.

Palabras clave: Armas biológicas, potencialidad, amenaza, prevención, protocolos.

\section{ABSTRACT}

The biological weapons natural or synthetic, they are products generated from some alive organisms or derivative of their manipulation physical-chemistry.

Until recently time for the Colombians mercenaries attacks with microorganisms did not generate reason for preoccupation because historically they have been developed in distant latitudes in Latin America. The states and communities jeopardize in prolonged conflicts and the presence of the terrorism is a parallel.

This permanent situation created sophisticated techniques, which stimulated to reach more complex levels of technological and manipulation in this type of arms.

However, in this country and particularly during the last three decades, the internal violence, and the presence of the narcotics-guerrilla and the narcotics-paramilitarismo generated different forms of terrorism.

A new actions envolved and penetrate like: massive kidnappings, to make attacks to institutions, to harm the oil infrastructure and municipalities takings. Settling down, from those actions the cyclical alteration of the order, neutralized today by democratic security.

Now days the terrorism are incorporated the use of the biological weapons is a requirement from the government to created and design a new protocol to control against this type of attacks.

Key Words: Biological weapon, potential, Threat, Prevention, Protocols. 


\section{EL BIOTERRORISMO EN LA HISTORIA.}

El empleo de agentes biológicos como arma no es un concepto nuevo. La historia de su uso ofrece numerosos ejemplos aun antes del desarrollo de la microbiología. Existen evidencias que indican que culturas como las persas y romanas, contaminaban el agua potable de los pozos y acueductos de sus enemigos, arrojando en ellos animales muertos.

En el siglo VI A.C, los Asirios envenenaban los pozos enemigos con desechos pútridos.

Durante el siglo XIV en Kaffa (ahora Feodossia) en Ucrania, las fuerzas tártaras sitiaron la ciudad y obligaron a rendirse a sus habitantes, infectándolos intencionalmente con Yersinia pestis (peste bubónica) al lanzar catapultados sobre las murallas de la ciudad, los cuerpos sin vida de sus propios soldados muertos por la plaga. Esta enfermedad epidémica causó el aniquilamiento de un tercio de la población europea de la época. Similar estrategia fue utilizada posteriormente por el ejército ruso contra el territorio Sueco en 1710.

En 1532 en la conquista de Perú, Francisco Pizarro repartió entre los Incas ropa (frazadas) de enfermos afectados y de muertos por viruela, así consiguió la rápida diseminación del virus, diezmando trágicamente a la población. Igualmente, en EEUU en 1763, Sir Jeffrey Amherst, comandante de las tropas británicas durante la guerra de independencia de Estados Unidos, diseminó este mismo virus entre los indígenas americanos utilizando a diferencia de Pizarro, pañuelos y cobijas infectados.

En 1940, se reportó con características endémicas, la presencia de una plaga en Manchuria y China, que posteriormente cobró forma epidémica, su aparición estuvo relacionada con una «lluvia de pulgas» infectadas con Yersinia pestis arrojadas por aviones japoneses que sobrevolaron la zona afectada.

Han transcurrido treinta y ocho años «desde que la Organización Mundial de la Salud (OMS) publicó el informe de 1970 (Health aspects of chemical and biological weapons (Aspectos de la salud en las armas químicas y biológicas)» ", desde entonces se han presentado importantes cambios en la teoría, los métodos y las experiencias de manipulación de microorganismos. En este sentido pueden mencionarse resultados obtenidos en procesos de investigación científica y aquellos capitalizados desde accidentes y situaciones casuales, como lo ocurrido por ejemplo durante 1979, cuando en las instalaciones de unos laboratorios microbiológicos, situados en la población de Sverdlovsk, se presentó una falla en los sistemas de filtración de aire internos,

${ }^{1}$ Afirmación del Secretario General de las Naciones Unidas ante la Asamblea General el 12 de octubre de 1998, documento. A/C.1/53/PV.3, 3-5. 
causando la diseminación de esporas de ántrax y provocando la muerte de 66 personas. De esta trágica experiencia se deriva la no menos fatal aparición de los primeros aerosoles infectantes. También en 1984, se registró otro de los múltiples episodios que caracterizaron esa ominosa década. Durante un solo mes en Dallas, Oregon, 751 personas desarrollaron gastroenteritis por Salmonella, luego de la contaminación a los «salad bar» de varios restaurantes en la ciudad de lo cual se responsabilizó a terroristas del culto religioso Rajneeshees.

En años posteriores otras novedosas y perversas estrategias fueron implementadas para causar pánico, en 2001 se convirtió en práctica frecuente la diseminación de esporas de ántrax, a través del servicio postal de los Estados Unidos, con la muerte directa de cinco personas, aumentó el temor ante ataques masivos similares.

Brevemente, debe recordarse que los atentados directos causaron estragos físicos, pero que son inmedibles y mayormente perdurables los grados de ansiedad y angustia infligidos a la población.

Debido a la magnitud de los eventos y a la escalada terrorista mundial, los organismos internacionales de control político y administrativo, orientaron de manera extraordinaria recursos financieros y de logística militar, para la creación de nuevos protocolos de defensa y para promocionar agendas legislativas en los países miembros. Se fortalece así y cobra renovada vigencia la Convención sobre Armas Biológicas y Toxinas (CAB) (Biological and Toxin Weapons Convention, $B W C$ ), firmada en 1972; para los ochenta, el documento recogía los riesgos de amenaza permanente de guerra biológica latentes, de acuerdo con las revelaciones hechas a comienzos de la década sobre los programas de armas biológicas de la antigua Unión Soviética e Irak, esto condujo a que los estados miembros establecieran un grupo ad hoc, con el mandato de negociar protocolos para reforzar la adhesión a la CAB y a la legalización de los proyectos de verificación sobre producción de este tipo de armas. Eventualmente, el esfuerzo de cooperación intercontinental se debilitó en el 2001 y el trabajo hacia la creación de un protocolo mundial se suspendió, prevaleciendo los proyectos y los protocolos de orden regional.

En mayo de 2001, la quincuagésima cuarta Asamblea Mundial de la Salud, en la resolución WHA54.14, le solicitó al Director General «brindar apoyo técnico a los Estados Miembros para el desarrollo y fortalecimiento de las actividades de preparación y respuesta contra los riesgos representados por los agentes biológicos, como una parte integral de los programas de manejo de emergencias»². Un año más tarde, en la resolución WHA55.16, la Asamblea solicitó al Director General «continuar con la expedición internacional de guías e información técnica sobre las medidas de salud pública, recomendadas para afrontar el uso deliberado de agentes biológicos y químicos que causan daño»². Más tarde la segunda edición del informe de 1970 se publicó en respuesta a estas solicitudes de la Asamblea Mundial de la Salud.

\footnotetext{
2 Afirmaciones del director general de la asamblea mundial de la salud. Fifty-fourth World Health Assembly, resolution WHA54.14, 21 May 2001, resolution WHA55.16, 18 May 2002.
} 
La amenaza del uso de armas biológicas por parte de las fuerzas armadas de los Estados involucrados, cambió sustancialmente desde el informe de 1970 y actualmente, es una preocupación latente en regiones del mundo donde numerosos países (Rusia, Japón, Estados Unidos, entre otros), todavía no se han adherido a las dos convenciones. Se suma al riesgo, que organizaciones particulares fuertemente financiadas han ido accediendo a la producción masiva de estas armas.

\section{PATÓGENOS DE INTERÉS EN BIOTERRORISMO.}

La clasificación que a continuación se expone, tiene como fuente la información de los Centers for Disease Control and Prevention (CDC), que determinan los posibles agentes infecciosos y de bioterrorismo. En dicha lista (cuadro1) se incluyen tres categorías en función de la facilidad de su transmisión y mortalidad producida.

\begin{tabular}{|c|c|c|}
\hline CATEGORIA A & CATEGORIA B & CATEGORIA C \\
\hline $\begin{array}{l}\text {-Se diseminan fácilmente o se } \\
\text { transmiten persona persona. } \\
\text {-Producen alta mortalidad, con } \\
\text { potencial para un gran impacto en } \\
\text { Salud pública. } \\
\text {-Pueden causar pánico y } \\
\text { desequilibrio social. } \\
\text {-Requieren acción especialmente, } \\
\text { intervención sanitaria pública. }\end{array}$ & $\begin{array}{l}\text {-Se diseminan con facilidad } \\
\text { moderada. } \\
\text {-Causan morbilidad moderada y } \\
\text { mortalidad baja. } \\
\text {-Requieren refuerzos específicos } \\
\text { de la capacidad diagnostica y } \\
\text { aumento en la vigilancia de la } \\
\text { enfermedad. }\end{array}$ & $\begin{array}{l}\text {-Son fácilmente disponibles. } \\
\text {-Presentan facilidad en su } \\
\text { producción y diseminación } \\
\text {-Poseen potencial para provocar } \\
\text { alta morbilidad y mortalidad, } \\
\text { además de tener impacto público } \\
\text { importante. }\end{array}$ \\
\hline
\end{tabular}

Luadro I. se presentan ıas caracterıstıcas mas ımportantes de cada una de ias categorıas de microorganısmos potencialmente peligrosos.

Tomado de: Centers for Disease Control and Prevention (2005). Potential Biological Weapons Threats. Bioterrorism.

Se mencionan igualmente los microorganismos que se incluyen en cada una de esas categorías (cuadro 2); es importante aclarar que en la categoría B se encuentran los microorganismos que 3 tienen una importante connotación frente a la seguridad de los alimentos y el agua. 
Cuadro 2

\begin{tabular}{|c|c|}
\hline \multirow[t]{6}{*}{ CATEGORIA A } & PATOGENO \\
\hline & $\begin{array}{l}\text { Bacillus anthracis (ántrax) } \\
\text { Clostridium botulinum toxin (botulismo) }\end{array}$ \\
\hline & Fiebres hemorrágicas virales \\
\hline & Francisella tularensis (tularemia) \\
\hline & Variola major (viruela) \\
\hline & Yersinia pestis (peste) \\
\hline \multicolumn{2}{|l|}{ CATEGORIA B } \\
\hline & Coxiella burnetti \\
\hline & Brucella species (brucelosis) \\
\hline \multirow[t]{9}{*}{ CATEGORIA B } & Rickettsia prowazekii (tifus) \\
\hline & AMENAZA CONTRA SEGURIDAD ALIMENTOS Y AGUA: \\
\hline & Burkholderia mallei (muermo) \\
\hline & Burkholderia pseudomallei (Melioidosis) \\
\hline & Chlamvdia psittaci (psitacosis) \\
\hline & Clostridium perfringens (toxina epsilon) \\
\hline & Crvptosporidium parvum \\
\hline & Enterobacterias: Salmonella, Shigella, Escherichia coli (O157:H7) \\
\hline & $\begin{array}{l}\text { Coxiella burnetti (Fiebre Q) } \\
\text { Vibrio Cholerae (Cólera) }\end{array}$ \\
\hline \multirow[t]{8}{*}{ CATEGORIA C } & Arbovirus (Dengue) \\
\hline & Flavovirus (Fiebre Amarilla) \\
\hline & Hantavirus \\
\hline & Leishmania (Leishmaniasis) \\
\hline & Mycobacterium Tuberculosis (Tuberculosis multiresistente). \\
\hline & Tick borne virus (Encefalit is) \\
\hline & Trypanosoma cruzi (Chagas) \\
\hline & Virus Nipah \\
\hline
\end{tabular}

Cuadro 2. Microorganismos potencialmente amenazantes.

Tomado de: Centers for Disease Control and Prevention (2005). Potential Biological Weapons Threats. Bioterrorism.

Las técnicas de modificación genética aplicadas a partir del primer ADN «recombinante» desarrollado en laboratorio, así como otros avances particulares de la biología molecular, aumentan el riesgo de producción de nuevos agentes para esta guerra. El acceso a los agentes biológicos se ha incrementado sustancialmente también a partir de los avances en microbiología industrial y por la experimentación militar.

En Colombia, recientes operaciones de desmantelamiento a organizaciones delictivas, han dejado en claro la existencia de siniestras redes internacionales, a las que se encuentran vinculados 
profesionales dedicados al terrorismo, quienes como parte de su entrenamiento bélico ofrecen entre otros, conocimientos y técnicas para la diseminación infectante de microorganismos, que resultan, bajo las condiciones biogeográficas del país, en un ambiente ideal para su aplicación. Esto puede corroborarse si se tienen en cuenta la fácil contaminación de fuentes de agua ubicadas en terreno montañoso a lo largo de nuestras tres cordilleras. El panorama general se complica además con el descubrimiento de la negociación de armas y elementos no convencionales (uranio), lo que aproxima otra dimensión al problema criminal latente. Aunque en menor escala, también se considera riesgo, el difícil control de ingreso de armas biológicas a través de las extensas fronteras.

A pesar del actual e incontrovertible éxito de la fuerza pública y de su importante presencia estratégica en el país, las razones anteriormente expuestas determinan, de forma inaplazable, incorporar a la política de seguridad nacional, el diseño e implementación de protocolos sobre sistemas de vigilancia, prevención y bioseguridad, que neutralicen los niveles de vulnerabilidad de ciudades y pueblos Colombianos frente al terrorismo, hoy, desafortunadamente articulado internacionalmente.

En coherencia con lo expuesto, la Universidad Militar Nueva Granada, adelanta actualmente procesos investigativos, que conducirán al diseño de protocolos de bioseguridad contra ataques terroristas, estructurados principalmente en los siguientes puntos:

\section{PREPARACIÓN Y RESPUESTA EN SALUD PÚBLICA.}

La respuesta inicial, a una liberación intencional de agentes infecciosos o tóxicos dirigida contra la población civil, es principalmente una responsabilidad local en muchas partes del mundo. Las autoridades locales están en la mejor posición para enfrentar tales eventos y serán quienes deban rendir cuentas, si el incidente no se maneja en forma adecuada. Mientras que los recursos nacionales e internacionales son importantes a largo plazo, es responsabilidad de las gobiernos locales garantizar que los sistemas y planes de respuesta se encuentren a punto antes de que se presente realmente un incidente (CEP, 1999).

\section{PREPARACIÓN.}

\subsection{Análisis de las amenazas.}

El análisis de amenazas es una actividad multidisciplinaria, a la que contribuyen las autoridades encargadas de hacer cumplir las leyes, los organismos de inteligencia y la comunidad médica y científica del país. Su propósito es identificar a quienes puedan tener intención de utilizar armas biológicas contra la población, los agentes que puedan ser usados y las circunstancias bajo las cuales se pueden usar. 
Este ejercicio es de amplio alcance y requiere enlaces activos entre las agencias que hacen cumplir las leyes, las de seguridad, las sanitarias (típicamente instituciones estatales centralizadas) y las autoridades locales. Tan sólo en raras oportunidades será posible identificar la probabilidad o la naturaleza precisa de la amenaza y por tanto, usualmente se requerirán medidas generales de preparación. La evaluación debe basarse en el análisis general de las circunstancias nacionales o locales. Aún si no se pueden identificar los peligros biológicos específicos, las mejoras generales en salud pública automáticamente incrementan la capacidad de una población para manejar los incidentes biológicos.

Si se pueden identificar amenazas potenciales específicas, se debe evaluar tanto la probabilidad de que suceda un accidente como sus consecuencias (CEP, 1999).

\subsection{Medidas de anticipación del ataque.}

El establecimiento de un sistema de respuesta biológico, es en sí mismo una estrategia de anticipación para la reducción del riesgo. Los antecedentes históricos sugieren que el riesgo de un ataque biológico se reduce considerablemente, con la sola existencia de una capacidad efectiva de respuesta y manejo del incidente. Si un agresor sabe que un ataque será rápido y eficientemente enfrentado, el incentivo para perpetrarlo se verá considerablemente disminuido. Es necesario llegar a un equilibrio entre el nivel de visibilidad de tal sistema de vigilancia y respuesta y, los resultados potencialmente negativos que ocurren tras la preocupación sobre posibles vulnerabilidades (UNISDR, 2002).

\subsection{Preparación para la respuesta.}

A pesar de los esfuerzos de anticipación, el riesgo de un ataque biológico no se puede eliminar completamente y podría acarrear serias consecuencias, si se llegase a presentar. Por tanto, se requiere un programa de preparación que contemple la adquisición de equipos y suministros, el desarrollo de procedimientos apropiados y el entrenamiento. Las comunidades deben examinar los protocolos existentes sobre materiales peligrosos, los planes de salud pública y el entrenamiento de la policía, los bomberos, el personal médico de urgencias y de salud pública, incluso médicos, epidemiólogos, veterinarios y personal de laboratorio. Todos ellos tendrán

que capacitarse sobre las características únicas de los agentes biológicos liberados intencionalmente (Leonard, 2000).

\subsection{Preparación de estrategias de comunicación e información pública.}

Se debe diseñar un plan para proporcionar información al público, mucho antes de que se presente un incidente y así, eliminar los mitos existentes sobre las armas biológicas. Para que la 
respuesta sea efectiva, el público necesita saber cómo debe actuar en caso de un ataque, mucho antes de que éste se presente. El plan de comunicaciones puede incluir transmisiones por radio, televisión y la distribución de folletos en los que se describa sin emotividad innecesaria, en lenguaje sencillo y directo la amenaza potencial. Se debe informar con claridad cómo se va a hacer pública la alarma y qué se debe hacer si sucede (Leonard, 2000).

\subsection{Validación de las capacidades de respuesta.}

Como sucede en la preparación para incidentes de grandes consecuencias pero baja frecuencia, probar y validar la capacidad de respuesta es un reto importante. Los simulacros realistas de entrenamiento son una herramienta útil y se deben evaluar críticamente para identificar los aspectos susceptibles de mejorar (APIC, 1999).

\section{RESPUESTA.}

\subsection{Respuesta previa a cualquier liberación de un agente biológico.}

Si se recibe una alerta sobre liberación inminente de agentes biológicos, se puede y se debe llevar a cabo una serie de actividades antes de que ésta suceda realmente. La secuencia en la cual se realicen estas actividades, dependerá de las circunstancias particulares del incidente. La primera indicación de un incidente puede ser la advertencia o el hallazgo de un artefacto inusual, o de materiales extraños como resultado de actividades normales de la comunidad, tales como la respuesta ante un incendio o el descubrimiento de un paquete extraño. Será necesario, entonces, llevar a cabo una o más de las siguientes acciones: (APIC, 1999).

- Análisis de la información disponible.

- Iniciación de un procedimiento de búsqueda.

- Establecimiento de un cordón.

- Identificación temprana de la naturaleza del peligro.

- Reducción o neutralización del riesgo.

\subsection{Respuesta a los incidentes biológicos.}

El cuadro 3, resume las principales actividades involucradas en la respuesta a los incidentes biológicos. La secuencia de eventos se basa en la aplicación de los principios de análisis de riesgo internacionalmente aceptados (UNISDR, 2002). 
Cuadro 3

\begin{tabular}{|c|c|}
\hline Evaluar los riesgos & $\begin{array}{l}\text { - Determinar si se ha presentado una liberación o si se esta } \\
\text { presentando un brote. } \\
\text { - Determinar la naturaleza del agente implicado y desarrollar una } \\
\text { definición del caso. } \\
\text { - Evaluar el potencial de diseminación del brote y los requerimientos } \\
\text { inmediatos y a largo plazo para manejo de casos, teniendo en cuenta } \\
\text { la posibilidad de que la infección sea contagiosa. }\end{array}$ \\
\hline $\begin{array}{l}\text { Manejo de los riesgos } \\
\text { (introducción a las medidas de } \\
\text { reducción / control de riesgos) }\end{array}$ & $\begin{array}{l}\text { - Proteger a los socorristas y a los trabajadores de la salud. } \\
\text { - Introducir los procedimientos de prevención y control de } \\
\text { infecciones. } \\
\text { - Conducir el triage de los casos. } \\
\text { - Garantizar el cuidado médico de los casos infectados. }\end{array}$ \\
\hline $\begin{array}{c}\text { Supervisar todas las } \\
\text { actividades }\end{array}$ & $\begin{array}{l}\text { - Decidir si los recursos locales y nacionales son adecuados o si se } \\
\text { debe buscar ayuda internacional. } \\
\text { - Implementar la vigilancia activa para supervisar la eficacia de los } \\
\text { procedimientos de prevención y control. } \\
\text { - Repetir el proceso de evaluación / manejo del riesgo según } \\
\text { requerimientos. } \\
\text { - Implementar las actividades de seguimiento a largo plazo. }\end{array}$ \\
\hline Comunicar los riesgos & $\begin{array}{l}\text { - Aplicar una estrategia de comunicación de riesgos para la } \\
\text { población afectada que tramita la información y las instrucciones } \\
\text { según la necesidad. }\end{array}$ \\
\hline
\end{tabular}

Cuadro 3. Principales actividades de respuesta a un ataque biológico.

Tomado de: UNISDR. United Nations International Strategy for Disaster Reduction. July 2002. (http:// www.unisdr.org).

\subsection{Principios del análisis de riesgos.}

La respuesta a los ataques biológicos es una tarea multidisciplinaria y compleja. Con tal número de temas y preguntas, se requiere contar con un medio para ordenar y priorizar el abordaje de la respuesta. Las actividades de respuestas requeridas y una secuencia ordenada lógicamente para su implementación, se pueden identificar al usar la aproximación del análisis de riesgos. Ésta es una forma organizada con la cual se identifican y evalúan las condiciones peligrosas y se pueden emprender acciones para eliminar, reducir o controlar los riesgos que representan. Estos pasos sirven para estructurar la planificación e identificar las áreas que necesitan atención durante la fase de "preparación» antes del ataque y la fase de «respuesta» posterior a la alarma o al ataque (Green, 2002).

\subsection{Principios de protección.}

Son diversas las tecnologías y estrategias de protección física contra la contaminación de agentes biológicos. En efecto, la protección individual es a menudo la medida que primero viene a la mente, al considerar los métodos para contrarrestar las amenazas biológicas. Sin embargo, la 
protección no se logra sin pagar un precio. El uso de ropa protectora es siempre un canje entre la protección lograda y los problemas causados por el equipo mismo. Por consiguiente, es un error considerar la protección aisladamente, debe ser vista como parte integral del proceso de manejo del riesgo, después de considerar las estrategias que lo pueden reducir y de descartarla por completo (Fraser, 2001).

\section{Vigilancia.}

Las siguientes son recomendaciones generales para la protección de grupos interdisciplinarios responsables de la seguridad nacional. Si durante un operativo de rutina (como el control y vigilancia de frontera marítima o terrestre), se debe proceder a requisar, individuos o material sospechoso, se deben tener en cuenta con anticipación las siguientes recomendaciones:

- Uso de los elementos de protección personal.

- Normas generales de bioseguridad para revisión y manipulación de material biológico.

- Material biológico sospechoso (caso kamikaze biológico).

- Comunicación y reporte.

- Rotulación y transporte de muestras biológicas.

\section{RED DE LABORATORIOS DE RESPUESTA PARA MONITOREAR ATAQUES BIOTERRORISTAS.}

Para incrementar la vigilancia y asistir a los laboratorios en su preparación para hacer frente a los eventos de bioterrorismo, el Centro para el Control de Enfermedades y prevención (CDC), ha propuesto una Red de Laboratorios de Respuesta (RLR) contra el bioterrorismo, la cual puede ser implementada en cualquier país. La red consiste de 3 niveles de laboratorios (figura 1), con diferentes capacidades de detección e identificación de agentes biológicos.

\subsection{Laboratorios Centinelas.}

Corresponden al primer nivel, son laboratorios clínicos clásicos. Ellos son la línea frontal para las víctimas de bioterrorismo, ya que estos pacientes visitan en primer lugar las clínicas, hospitales y cuartos de urgencias. Este tipo de laboratorio debe tener la capacidad de detectar posibles agentes y/o descartar otros, así como enviar a un laboratorio de nivel de referencia los aislamientos sospechosos. De estos laboratorios depende la detección temprana de los agentes biológicos.

\subsection{Laboratorios de Referencia.}

Están en el segundo nivel y ofrecen exámenes para confirmar la identificación y caracterizar la sensibilidad de los agentes a los antibióticos. Corrientemente estos laboratorios adelantan exámenes, que incluyen anticuerpos por fluorescencia directa, pruebas de aglutinación, entre 
otros. Deben contar al menos con facilidades de BSL-2 de bioseguridad. En adición, deben tener personal entrenado para realizar pruebas de PCR y manejo de cabinas de seguridad BSL3. Deben trabajar en estrecha relación con los laboratorios centinelas y ofrecer consultoría en protocolos de identificación y bioseguridad.

\subsection{Laboratorio Nacional.}

Es el último nivel en la red y le corresponde confirmar cualquier aislamiento, además concluye investigaciones iniciadas por los laboratorios de referencia. Debe contar con facilidades de nivel BSL-3 de bioseguridad. Es de su competencia informar a las autoridades la confirmación de casos de bioterrorismo.

De más está decir que los miembros de los equipos de laboratorio involucrados en la red, deben estar muy bien preparados en la detección e identificación de los potenciales agentes de bioterrorismo, a través de constante entrenamiento, cursos de afianzamiento en microbiología , biotecnología y ejercicios de preparación sin previo aviso.

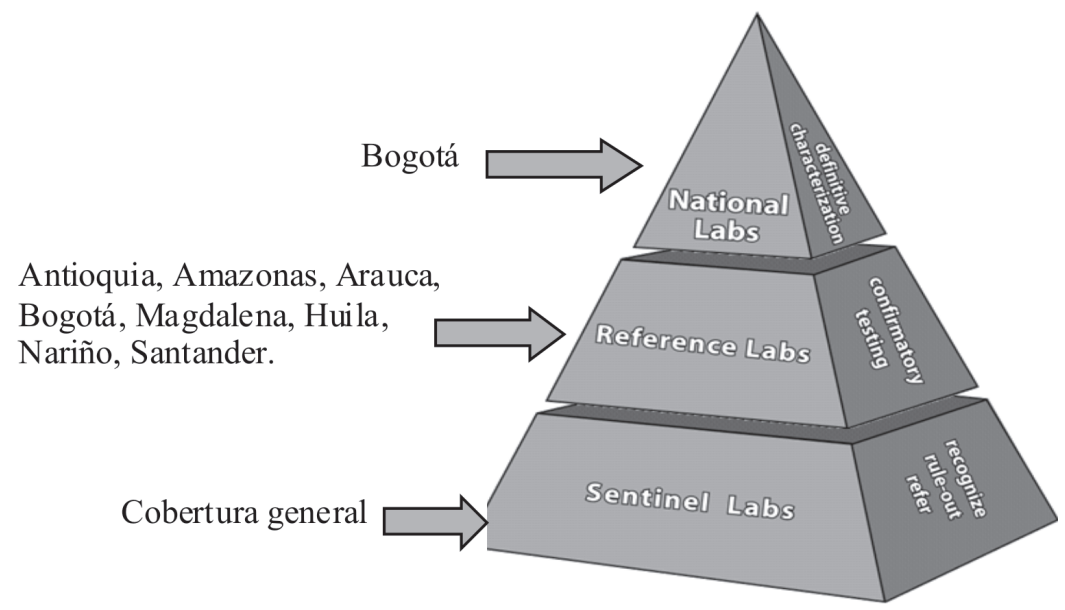

Figura 1. Red de laboratorios de Colombia. Laboratorio nacional (Bogota), laboratorios de referencia (Antioquia, Amazonas, Arauca, Bogota, Magdalena, Huila, Nariño, Santander, Valle).

Tomado de: Centers for Disease Control and Prevention (2005). Potential Biological Weapons Threats. Bioterrorism.

El incremento en las comunicaciones, la tecnología actual y la introducción de nuevos métodos de diagnóstico, hacen posible una coordinación más eficiente de la red de laboratorios para detectar, reportar y responder ante cualquiera enfermedad infecciosa, incluso, no necesariamente de bioterrorismo. En éste sentido, la introducción de los sistemas computarizados de identificación, han significado un paso adelante en la vigilancia moderna de éstos agentes. 


\section{CONCLUSIONES.}

Con el objeto de optimizar la capacidad de reacción ante posibles ataques bioterroristas, las instituciones encargadas de la defensa nacional, deben optimizar actualmente su sistema estratégico y de seguridad, mediante la incorporación del protocolo de bioseguridad.

La habilidad para reaccionar eficaz y oportunamente ante la ofensiva con armas biológicas, depende del conocimiento y el entrenamiento de los grupos interdisciplinarios responsables de la seguridad nacional.

\section{GLOSARIO}

DNA recombinante: Cualquier molécula de DNA formada por la unión de segmentos de DNA procedentes de diferentes fuentes. Los DNA recombinantes son ampliamente utilizados en el clonaje de genes, en la modificación genética de organismos y en biología molecular en general.

Aglutinación: Es un método de laboratorio para examinar ciertos anticuerpos o antígenos en una variedad de fluidos corporales, como la saliva, la orina, el líquido cefalorraquídeo o la sangre.

BSL-2/3: En función del nivel de riesgo biológico, un laboratorio puede clasificarse según el denominado Nivel de Bioseguridad:

BSL-2: nivel aplicable a laboratorios con fines clínicos, de diagnóstico, etc., donde existen agentes de riesgo moderado pero no se transmiten vía aerosol.

BSL-3: en este nivel existe riesgo de transmisión por vía respiratoria de infecciones potencialmente mortales. Deben considerarse barreras de protección. Todas las manipulaciones deben llevarse a cabo en Cabinas de Seguridad Biológica. Conducir el triage de los casos.

Morbilidad: Se refiere a los efectos de una enfermedad en una población en el sentido de la proporción de personas que la padecen en un sitio y tiempo determinado. Epidemiológicamente, se puede ampliar al estudio y cuantificación de la presencia y efectos de una enfermedad en una población.

Fluorescencia directa: Es una técnica de laboratorio, que permite entre otros usos, marcar anticuerpos que se fijarán a los antígenos específicos, permitiendo la identificación del microorganismo contra el cual se formaron. 
PCR (Reacción en cadena de la polimerasa): Técnica que permite amplificar determinadas regiones de DNA, mediante múltiples ciclos de polimerización de DNA, cada uno de los cuales es seguido por un breve tratamiento de incremento de temperatura para separar las cadenas complementarias.

Triage: Método que permite clasificar, agrupar o seleccionar la naturaleza de un caso, dependiendo del grado de emergencia.

\section{BIBLIOGRAFIA}

- $\quad$ APIC/CDC, Bioterrorism readiness plan (1999). A template for healthcare facilities.

- $\quad$ APIC. Bioterrorism Task Force (Judith F. English, Mae Y. Cundiff, John D. Malone and Jeanne Pfeiffer) and CDC Hospital Infections Program Bioterrorism Working Group. (Michael Bell, Lynn Steele and Michael Miller).

- $\quad$ Centers for Disease Control and Prevention (CDC). (2005). Potential Biological Weapons Threats. Bioterrorism.

- $\quad$ CDC- Biological and Chemical Terrorim. (2000). Strategic Plan for Preparedness and Response. Recommendations of the CDC strategic planning workgroup. MMWR; 49 (RR04); 1-14.

- $\quad$ CEP. Community emergency preparedness (1999). A manual for managers and policy-makers. Geneva, World Health Organization.

- $\quad$ Fraser MR, Fisher VS (2001). Elements of effective bioterrorism preparation: a planning primer for local public health agencies. Washington, DC, National Association of County and City Health Officials.

- $\quad$ Green M, Kaufman Z (2002). Surveillance for early detection and monitoring of infectious disease outbreaks associated with bioterrorism. Israel Medical Association Journal. 4:503-506.

- Klietmann, F., and K. L. Ruoff. (2001). Bioterrorism: implications for the clinical microbiologist. Clin. Microbiol. Rev. 14:364-381

- $\quad$ Lareo, L. (2005). Memorias del Diplomado en Microbiología Forense y Bioterrorismo. Principios de Bioterrorismo. Pontificia Universidad Javeriana.

- $\quad$ Leonard B. (2000). Emergency response guidebook: a guidebook for first responders during the initial phase of a dangerous goods/hazardous materials incident. Ottowa, ON, Diane Publishing Co.

- Millar, J. Engelberg S and Broad W. (2002). Guerra bacteriológica. Ediciones B, S.A Barcelona, España.

- $\quad$ Políticas de Prevención del Terrorismo (2003). Departamento de Estado, Washington D.C.

- $\quad$ UNISDR. United Nations International Strategy for Disaster Reduction. (2002). Living with risk: a global review of disaster reduction initiatives. 